Ex, P.; Busse, R.; Henschke, C.

\title{
Die Nutzenbewertung von nichtmedikamentösen Untersuchungs- und Behandlungsmethoden
}

Welche Tragweite hat die Regelung nach § 137h SGB V?

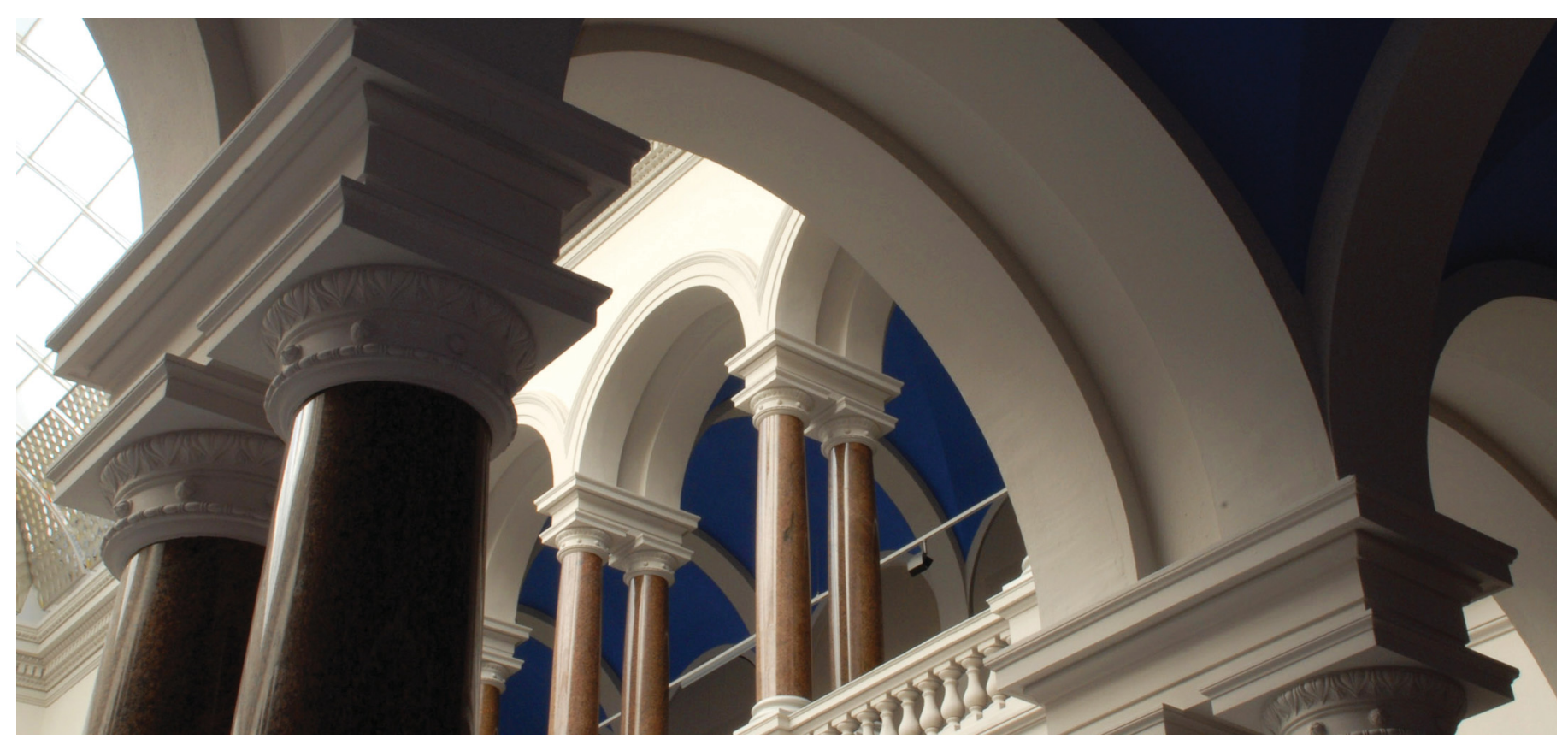

Ex, P., Busse, R., \& Henschke, C. (2016). Die Nutzenbewertung von nichtmedikamentösen Untersuchungs- und Behandlungsmethoden. Gesundheits- Und Sozialpolitik, 70(2), 48-55. https://doi.org/10.5771/1611-5821-2016-2-48 
Patricia Ex, Reinhard Busse, Cornelia Henschke

\section{Die Nutzenbewertung von nichtmedikamentösen Untersuchungs- und Behandlungsmethoden: Welche Tragweite hat die Regelung nach § 137h SGB V?}

Kontaktanschrift:

Patricia Ex

Fachgebiet Management im Gesundheitswesen

+493031428420

patricia.ex@campus.tu-berlin.de

Patricia Ex ist Doktorandin am Fachgebiet Management im Gesundheitswesen an der TU Berlin und Senior Consultant bei WMP HealthCare $\mathrm{GmbH}$

Prof. Dr. med. Reinhard Busse ist Leiter des Fachgebietes Management im Gesundheitswesen an der TU Berlin und des Gesundheitsökonischen Zentrums Berlin (Berlin HECOR) sowie Associate Director for Research Policy des European Observatory on Health Systems and Policies

Dr. rer. oec. Cornelia Henschke ist wissenschaftliche Mitarbeiterin am Fachgebiet Management im Gesundheitswesen an der TU Berlin sowie Koordinatorin des Gesundheitsökonomischen Zentrums Berlin (Berlin HECOR) 


\section{Anlesetext}

Mit dem § 137h SGB V wurde erstmals eine Nutzenbewertung im stationären Sektor eingeführt. Bestimmte neue Untersuchungs- und Behandlungsmethoden (NUB), die maßgeblich auf der Anwendung eines Medizinproduktes mit hoher Risikoklasse beruhen, werden zukünftig einer Nutzenbewertung unterzogen. Dabei bestehen einige Einschränkungen und offene Punkte in der Regelung, die es zu diskutieren gilt. Ziel dieses Beitrages ist, die Neuregelung im gesundheitspolitischen und gesundheitswissenschaftlichen Kontext darzustellen und ihre Tragweite für das deutsche Gesundheitssystem zu diskutieren.

Nutzenbewertung Medizinprodukte, Neue Untersuchungs- und Behandlungsmethoden (NUBs), Vergütung von Innovationen, GKV-Leistungskatalog 


\section{Einführung}

Die Gesundheitsversorgung wird durch neue Therapieansätze und Diagnoseverfahren unter Einsatz von Arzneimitteln und Medizinprodukten stetig fortentwickelt. Mit Ausgaben der Bundesrepublik für Forschung und Entwicklung im Bereich Gesundheit von rund zwei Milliarden Euro im Jahr 2015 gilt das Gesundheitswesen als eine der innovativsten Wirtschaftsbranchen Deutschlands. ${ }^{1}$ Dabei tragen neue oder weiter entwickelte Gesundheitstechnologien - also Arzneimittel, Medizinprodukte und -geräte ebenso wie Behandlungsmethoden - u. a. zu Verringerungen von Nebenwirkungen, Verkürzungen von Operations- und Heilungsdauern, genaueren Diagnosestellungen sowie zur Steigerung der Wirtschaftlichkeit und der Lebensqualität bei. ${ }^{2}$

Als problematisch erweist sich jedoch, dass zum Zeitpunkt der Zulassung neuer Gesundheitstechnologien oft der genaue Nutzen und mögliche Risiken bei breiter Anwendung eines Produkts unklar sind. Als "neu“ werden - der leistungsrechtlich orientierten Definition folgend - alle Gesundheitstechnologien verstanden, die nicht in den Leistungskatalogen der GKV gelistet sind. ${ }^{3}$ Insbesondere in einem solidarisch finanzierten System stellt sich die Frage, welche neuen Technologien unter welchen Voraussetzungen in die Erstattung aufgenommen werden. In diesem Kontext wurden in zahlreichen Gesundheitssystemen Institutionen und Prozesse etabliert, die eine evidenzbasierte Entscheidungsfindung unterstützen. Insbesondere werden nach erfolgter Zulassung systematische Bewertungen von Gesundheitstechnologien (Health Technology Assessments, HTA) durchgeführt, um mit diesen Informationen eine Grundlage für Entscheidungen im Gesundheitssystem zu schaffen. ${ }^{4,5}$ Das steht im Gegensatz zur klinischen Forschung, die aus Erkenntnisgründen im Rahmen der Zulassung durchgeführt wird. ${ }^{6}$

Während sich die Bewertung von Technologien prinzipiell seit den 90er Jahren in Deutschland verbreitet hat, gab es bis 2011 keine systematische, $d$. h. regelhaft stattfindende Bewertung des Nutzens der auf den Markt kommenden neuen Technologien. Seit Inkrafttreten des Arzneimittelmarktneuordnungsgesetzes (AMNOG) wird für neue erstattungsfähige Arzneimittel der Zusatznutzen verglichen mit einer zweckmäßigen Vergleichstherapie bewertet. Damit dürfen zugelassene Arzneimittel weiterhin umgehend im Gesundheitswesen angewandt werden, müssen aber zeitgleich die frühe Nutzenbewertung durchlaufen. Arzneimittel, die 
ausschließlich im stationären Sektor angewandt werden, sind von der Prüfung der frühen Nutzenbewertung ausgenommen. Dies entspricht der generellen Herangehensweise im stationären Sektor - dem „Verbotsvorbehalt“ - wonach alle Methoden im stationären Sektor zu Lasten der gesetzlichen Krankenkassen erbracht werden dürfen, solange sie nicht explizit vom G-BA verboten sind (§ 137c SGB V). Mit dem im Juli 2015 in Kraft getretenen Versorgungsstärkungsgesetz (VSG) hat der Gesetzgeber eine Nutzenbewertung im stationären Sektor eingeführt, und zwar von neuen Untersuchungs- und Behandlungsmethoden (NUB), die maßgeblich auf dem Einsatz eines Medizinproduktes hoher Risikoklassen beruhen (im Folgenden auch „Nutzenbewertung von Medizinprodukten“). Bestimmte Methoden, für die erstmalig ein NUB-Entgelt im stationären Sektor beantragt werden soll, müssen demnach zukünftig eine Nutzenbewertung durchlaufen.

Intention dieser Analyse ist, die Neuregelung zur Nutzenbewertung von Medizinprodukten hoher Risikoklassen im gesundheitspolitischen und gesundheitswissenschaftlichen Kontext darzustellen und ihre Tragweite im deutschen Gesundheitssystem zu diskutieren. Dafür werden zunächst die historische Entwicklung der Bewertung von Gesundheitstechnologien sowie eine Übersicht der geltenden Rahmenbedingungen zur Anwendung und Vergütung von neuen Gesundheitstechnologien dargestellt. Anschließend wird die neue Regelung aufgezeigt. Auf Grundlage der NUBs des Jahres 2016 wird diskutiert, in welchem Ausmaß die Regelung in der Praxis voraussichtlich Anwendung finden wird.

\section{Gesundheitswissenschaftlicher und regulatorischer Kontext}

\subsection{Die Entwicklung von Health Technology Assessments (HTA)}

Seit Veröffentlichung des ersten HTA-Berichts (Health Technology Assessment, dt. Bewertung von Gesundheitstechnologien) des U.S. Office of Technology Assessment (OTA) zu politischen Implikationen des Computertomographie (CT) Scanners im Jahr 1978 hat sich die Erstellung von HTA-Berichten zur Unterstützung von Erstattungsentscheidungen international etabliert. ${ }^{7}$ Ab Ende der 1980er Jahre wurden in zahlreichen Gesundheitssystemen Institutionen zur Durchführung von Health Technology Assessments gegründet. In Deutschland wurde HTA ab 1995 mit finanzieller Unterstützung politisch gefördert und mit dem GKV-Neuordnungsgesetz 
1997 gesetzlich verankert. So wurde der damalige Bundesausschuss für Ärzte und Krankenkassen beauftragt, neue und bestehende ambulante Technologien zu bewerten. ${ }^{8}$ Das HTA-Mandat wurde zusätzlich im Jahr 2000 auf das Deutsche Institut für Medizinische Dokumentation und Information (DIMDI) ausgeweitet. ${ }^{9}$

Nachdem HTA-Institutionen zunächst primär nicht-medikamentöse Technologien bewerteten, war das britische National Institute for Health and Care Excellence (NICE) 1999 die erste Institution, die neben nicht-medikamentösen Technologien auch Arzneimittel nach der Zulassung überprüft. ${ }^{10}$ Frankreich und Deutschland begannen 2004 mit dem Einschluss medikamentöser Technologien: In Frankreich wurde als Institution die Haute Autorité de Santé (HAS) gegründet, die für Bewertungen von Gesundheitstechnologien zuständig ist (bis 2004 als Agence nationale d'accréditation et d'évaluation en santé, ANAES). In Deutschland wurde im gleichen Jahr das Institut für Qualität und Wirtschaftlichkeit im Gesundheitswesen (IQWiG) mit der Maßgabe gegründet, als wissenschaftlich unabhängiges Institut neue Technologien für Entscheidungen des G-BA zu bewerten. In der aktuellen Praxis bestehen neben dem IQWiG zahlreiche Institutionen, die eigenständig Bewertungen von Gesundheitstechnologien vornehmen, wie unter anderem der Medizinische Dienst der Krankenkassen (MDK) sowie Abteilungen bei der Bundesärztekammer und der Kassenärztlichen Bundesvereinigung. Von den möglichen Schwerpunkten eines HTA-Berichts - der Bewertung von Sicherheit, Wirksamkeit bzw. Nutzen, Kostenwirksamkeit, sowie ethischer, organisatorischer, sozialer und legaler Implikationen ${ }^{11}$ - werden de facto insbesondere medizinische Effektivität und Wirtschaftlichkeit untersucht. ${ }^{12}$

Mit Nutzenbewertungen wird das Ziel verfolgt, unter Alltagsbedingungen den tatsächlichen Nutzen der Gesundheitstechnologie nach erfolgter Zulassung als Grundlage für Vergütungsentscheidungen zu überprüfen. Damit entsprechen sie prinzipiell den Kriterien eines HTA-Berichts. Von den möglichen Schwerpunkten wird hier der Nutzen einer Technologie untersucht. Der Begriff des Nutzens kann in diesem Kontext verstanden werden als „Effekte einer Intervention, die in mehr als geringfügigem Ausmaß eine Verbesserung der Prognose und/oder der Symptomatik/Lebensqualität von Patienten" zur Folge haben. ${ }^{13}$ Dabei ist weniger die Frage, wie eine Technologie wirkt, sondern ob und in welchem Ausmaß Betroffene einen gesundheitlichen Vorteil durch die Intervention im Vergleich zu einer anderen 
oder keinen Intervention haben. ${ }^{14,15}$ Damit entspricht der Nutzen einer bestimmten Form der Wirksamkeit, nämlich der Wirksamkeit unter Alltagsbedingungen (effectiveness) [im Gegensatz zur Wirksamkeit unter Studienbedingungen (efficacy)] und mit direkter Relevanz für den Patienten. ${ }^{13}$

Allerdings folgen Nutzenbewertungen nicht in allen Aspekten der Systematik eines HTA-Berichtes: Zum einen wird bei HTA-Berichten in der Regel als erster Teil eine systematische Literaturrecherche durchgeführt, um auf dieser Grundlage möglichst alle existierenden Unterlagen in die Bewertung einzubeziehen. Bei den bestehenden Verfahren der Nutzenbewertung von Medizinprodukten und Arzneimitteln hingegen werden die von Herstellern und Krankenhäusern eingereichten Studien bzw. Unterlagen auf ihre Plausibilität überprüft. Auch die individuelle Entscheidung darüber, welche der möglichen Technologien überprüft werden soll, ist ein zentrales Element von HTA, das in diesem Fall keine Anwendung findet.

Trotz ihrer Verbreitung werden HTA-Berichte im eigentlichen Sinne in Deutschland selektiv für einzelne Technologien durchgeführt. Einige Akteure, insbesondere Vertreter des GKV-Spitzenverbandes, des IQWiG und des Medizinischen Dienstes des Spitzenverbandes Bund der Krankenkassen (MDS), sprachen sich daher für eine systematische Untersuchung des Nutzens neuer Medizinprodukte nach deren Zulassung aus. ${ }^{16-18}$

\subsection{Anwendung und Erstattung von neuen Gesundheitstechnologien} im deutschen Gesundheitswesen

Die Zulassung von Medizinprodukten erfolgt im Rahmen eines Konformitätsbewertungsverfahrens bei einer Benannten Stelle in der Europäischen Union (EU). Bei erfolgreichem Erlangen eines CE-Kennzeichens darf das Produkt aufgrund des freien Warenverkehrs im gesamten europäischen Wirtschaftsraum vermarktet und verkauft werden. ${ }^{19}$ Im Gegensatz zur Zulassung sind Anwendungsund Erstattungsregelungen sowie eine ggf. damit verbundene Nutzenbewertung nationalstaatlich reguliert. Diese Regelungen unterscheiden sich in Deutschland abhängig von Produktgruppe (Arzneimittel oder Medizinprodukte wie Hilfsmittel, Großgeräte etc.) und Sektor, in dem ein Produkt angewandt wird. Im Folgenden liegt 
der thematische Schwerpunkt auf neuen Untersuchungs- und Behandlungsmethoden im stationären Sektor.

Insgesamt müssen Leistungen der GKV „ausreichend, zweckmäßig und wirtschaftlich sein; sie dürfen das Maß des Notwendigen nicht überschreiten“ (§ 12 SGB V). ${ }^{20}$ Dieses "Wirtschaftlichkeitsgebot" ist in Zusammenhang mit § 2 SGB V zu betrachten, in dem es heißt „Qualität und Wirksamkeit der Leistungen haben dem allgemein anerkannten Stand der medizinischen Erkenntnisse zu entsprechen und den medizinischen Fortschritt zu berücksichtigen“. ${ }^{21}$ Gemäß dem Prinzip des „Verbotsvorbehaltes“ dürfen Untersuchungs- und Behandlungsmethoden im Krankenhaussektor ohne systematische Bewertung ihrer Wirksamkeit angewandt werden. Nur solche Methoden, die zuvor vom G-BA explizit verboten wurden, sind von der Leistungserbringung zu Lasten der Krankenkassen im stationären Sektor ausgeschlossen (§ 137c SGB V).

Aufgrund des Sachleistungsprinzips und der solidarischen Finanzierung des deutschen GKV-Systems hängt die Verbreitung und breitflächige Anwendung von Gesundheitsleistungen eng mit deren Erstattung zusammen. Die Finanzierung der Betriebskosten im stationären Sektor erfolgt über Fallpauschalen (sog. DiagnosisRelated Groups, DRGs). Mit diesen werden fallbezogene Behandlungskosten in Abhängigkeit von Diagnose, Schweregrad der Erkrankung sowie durchgeführten Operationen und Prozeduren pauschal nach dem durchschnittlichen betrieblichen Aufwand vergütet. Damit sind Ausgaben für Personal- und Sachkosten (z. B. für Implantate) pauschal in der Vergütung enthalten. Aufgrund dieser Systematik geht der Sachverständigenrat zur Begutachtung der Entwicklung des Gesundheitswesens davon aus, dass neue Technologien, die günstiger sind als bestehende - und damit von der vorhandenen DRG abgedeckt sind - in das Gesundheitssystem gelangen, ohne dass dies in der Breite festzustellen ist. ${ }^{22}$ Teurere Gesundheitstechnologien benötigen hingegen eine Anpassung der Vergütungsziffern oder eine zusätzliche, separate Finanzierung.

Zwischen Einführung einer neuen Technologie und deren Integration ins DRGSystem nennt der GKV-Spitzenverband eine zeitliche Verzögerung von ca. drei Jahren. ${ }^{23}$ Um eine schnellere Vergütung und damit breitere Anwendung von neuen Technologien zu ermöglichen, gibt es in Deutschland, ebenso wie in den meisten Ländern mit DRG-System, zusätzliche temporäre Vergütungsinstrumente. ${ }^{24,25}$ In 
Deutschland sind das neben Vertragsmodellen der integrierten Versorgung, der Erprobungsregelung sowie bewerteten und unbewerteten Zusatzentgelten insbesondere Entgelte für Neue Untersuchungs- und Behandlungsmethoden (NUB) (§ 6 Abs. 2 KHEntgG). Mit dieser sogenannten „Innovationsklausel“ hat der Gesetzgeber Krankenhäusern und Krankenkassen auf Landesebene die Möglichkeit gegeben, zeitlich befristete Vergütungen für ärztliche Methoden, Arzneimittel und Medizinprodukte zu vereinbaren. Diese sogenannten NUB-Entgelte gelten jeweils nur für ein Jahr und nur für das beantragende Krankenhaus.

Um ein NUB-Entgelt für eine neue Technologie zu erhalten, sind beantragende Krankenhäuser verpflichtet, bis zum 31. Oktober beim Institut für das Entgeltsystem im Krankenhaus (InEK) anzufragen, ob die spezifische Technologie bereits im DRGSystem abgedeckt ist. Bis zum 31. Januar des darauffolgenden Jahres entscheidet das InEK, ob eine angemessene Vergütung im DRG-System für die Methode vorhanden ist. Das Ergebnis der Prüfung wird, ohne Angabe von Gründen, in vier Kategorien als Status 1-4 vergeben (s. Tabelle 1).

Tabelle 1: Prüfergebnisse für NUB-Entgelte durch das InEK

\begin{tabular}{|c|l|}
\hline Status 1 & $\begin{array}{l}\text { Kriterien der NUB-Vereinbarung sind erfüllt. } \\
\text { Für diese Methoden/ Leistungen ist die Verhandlung eines NUB-Entgeltes für } \\
\text { das entsprechende Jahr zulässig. }\end{array}$ \\
\hline Status 2 & $\begin{array}{l}\text { Kriterien der NUB-Vereinbarung sind nicht erfüllt. } \\
\text { Für diese Methoden/ Leistungen ist die Verhandlung eines NUB-Entgeltes für } \\
\text { das entsprechende Jahr nicht zulässig. }\end{array}$ \\
\hline Status 3 & Methode / Leistung konnte nicht in der festgesetzten Frist bearbeitet werden. \\
\hline Status 4 & $\begin{array}{l}\text { Informationen der Anfrage sind unplausibel oder nicht nachvollziehbar. In } \\
\text { begründeten Einzelfällen dürfen hierfür NUB-Entgelte verhandelt werden. }\end{array}$ \\
\hline
\end{tabular}

Quelle: in Anlehnung an NUB-Aufstellung des InEK ${ }^{26}$

Kommt das InEK zu dem Ergebnis, dass eine Methode nicht im DRG-System abgebildet ist (Status 1), darf jedes Krankenhaus, das eine Anfrage an das InEK gerichtet hat, mit den Krankenkassen auf Landesebene im Rahmen oder außerhalb der Budgetvereinbarungen für die angefragte Methode in Vergütungsverhandlungen treten. Die eingeführte Nutzenbewertung von Medizinprodukten nach § 137h SGB V ist an die Beantragung ebendieser NUB-Entgelte geknüpft. 


\section{Die Nutzenbewertung von Methoden mit Medizinprodukten hoher}

Risikoklassen

Mit dem Versorgungsstärkungsgesetz hat der Gesetzgeber eingeführt, dass der GBA für bestimmte neue Untersuchungs- und Behandlungsmethoden, die auf dem maßgeblichen Einsatz eines Medizinproduktes mit hoher Risikoklasse basieren und die im stationären Sektor angewandt werden sollen, eine Nutzenbewertung durchführt (§ 137h SGB V). Der Begriff der „Methode“ bezeichnet nach Rechtsprechung des Bundessozialgerichts - auf die auch der G-BA verweist - eine „medizinische Vorgehensweise, der ein eigenes theoretisch-wissenschaftliches Konzept zugrunde liegt, welches sie von anderen Verfahren unterscheidet und das ihre systematische Anwendung in der Untersuchung und Behandlung bestimmter Krankheiten rechtfertigen soll. “27 Eine Methode ist daher von einzelnen Medizinprodukten wie Instrumenten, Apparaten etc. für diagnostische und therapeutische Zwecke (§ $3 \mathrm{MPG}$ ) abzugrenzen.

Die Nutzenbewertung gilt prinzipiell für Methoden mit Medizinprodukten, die als Risikoklasse Ilb oder III oder als aktives implantierbares medizinisches Gerät definiert sind und für die im betreffenden Jahr erstmals ein NUB-Entgelt beantragt wird. ${ }^{28}$ Die Einteilung von Medizinprodukten nach Risikoklassen wird in Abhängigkeit von deren Zweckbestimmung während der Zulassung auf Grundlage von verschiedenen EU-Richtlinien vorgenommen: Klasse llb sind Medizinprodukte mit "hohem Risiko" wie Beatmungsgeräte, und Anästhesie- und Röntgengeräte. ${ }^{4}$ Medizinprodukte der Klasse III haben ein „sehr hohes Risiko“, z. B. Herzklappen, Koronarstents, künstliche Gelenke und Brustimplantate. ${ }^{29}$ Aktiven implantierbaren Geräten ist ebenfalls ein sehr hohes Risiko zuzuordnen. Sie können wie Klasse IV verstanden werden, sind jedoch in einer separaten EU-Richtlinie geregelt. Sie beinhalten u. a. Herzschrittmacher, implantierbare Defibrillatoren und CochleaImplantate. ${ }^{30}$

Eine Nutzenbewertung soll nur für Methoden mit solchen HochrisikoMedizinprodukten durchgeführt werden, die zusätzlich einen „besonders invasiven Charakter" haben. Damit lassen sich aus den Vorgaben der BMG-Verordnung sowie den entsprechenden EU-Richtlinien drei Produktgruppen von Medizinprodukten ableiten, die im Rahmen der Methode eine Nutzenbewertung durchlaufen werden: 


\section{- Medizinprodukte mit Risikoklasse IIb,}

die mittels Aussendung von Energie oder Abgabe radioaktiver Stoffe gezielt auf wesentliche Funktionen von Organen oder Organsystemen (insbes. Herz, zentrales Kreislaufsystem sowie zentrales Nervensystem) einwirken;

- Medizinprodukte mit Risikoklasse III, mit deren Anwendung die wesentlichen Funktionen von Organen oder Organsystemen (insbes. Herz, zentrales Kreislaufsystem sowie zentrales Nervensystem) langzeitig verändert oder ersetzt werden;

- „Aktive implantierbare medizinische Geräte“, die durch einen chirurgischen Eingriff eingesetzt werden, um nach dem Eingriff dort zu verbleiben und deren Betrieb von einer Stromquelle oder einer anderen Energiequelle abhängig ist.

Das gesamte Verfahren beginnt parallel mit der Anfrage für NUB-Entgelte beim InEK jährlich bis zum 31. Oktober (für eine visuelle Darstellung zum Ablauf des Verfahrens siehe das Schaubild in Abbildung 1). Mit dem Antrag für ein NUB-Entgelt muss das Krankenhaus für Methoden mit oben genannten Medizinproduktgruppen Informationen zum Stand der wissenschaftlichen Erkenntnis der Methode und zur Anwendung des Medizinprodukts an den G-BA übermitteln. Die Übermittlung erfolgt nicht auf Initiative, sondern im Benehmen mit dem Hersteller des Medizinprodukts.

Einer Nutzenbewertung werden ausschließlich solche Methoden unterzogen, die ein neues Wirkprinzip oder ein neues Anwendungsgebiet - ein sogenanntes neues theoretisch-wissenschaftliches Konzept - aufweisen. Nach Eingang der Informationen hat der G-BA zwei Wochen Zeit für alle Methoden zu überprüfen, ob es sich jeweils um ein neues theoretisch-wissenschaftliches Konzept handelt. Ist dem nicht so, kehrt die Methode in das reguläre Verfahren für NUB-Entgelte zurück. Handelt es sich laut G-BA um ein neues theoretisch-wissenschaftliches Konzept, werden alle Krankenhäuser, die die Methode anwenden wollen, sowie Medizinproduktehersteller um Zusendung weiterer Informationen innerhalb von vier Wochen aufgefordert. Mit einer dreimonatigen Frist hat der G-BA anschließend, unter regelmäßiger Beauftragung des IQWiG, eine Bewertung des Nutzens, Schadens und Potentials der Methode vorzunehmen. Aufgrund der genannten Fristen sind die Bewertungen ungefähr bis Mitte März zu erwarten. Parallel dazu läuft wie gehabt die NUB-Prüfung des InEK. Dessen Mitteilung, welche Methoden bislang nicht im DRG- 
System abrechenbar sind - für die prinzipiell ein NUB-Entgelt verhandelt werden darf - erfolgt während der Nutzenbewertung des G-BA jährlich bis zum 31. Januar.

Als Ergebnis der Nutzenbewertung durch den G-BA sind drei verschiedene Ausgänge möglich. Bestenfalls ist der Nutzen der Methode unter Anwendung des Medizinprodukts hinreichend belegt. Falls das InEK einen Status 1 vergibt, kann der G-BA Qualitätskriterien für die Leistungserbringung festlegen. Ein Entgelt ist innerhalb von drei Monaten nach dem Beschluss zwischen dem beantragenden Krankenhaus und den entsprechenden Krankenkassen auf Landesebene zu vereinbaren. Ansonsten kann die Schiedsstelle des jeweiligen Landes - gebildet aus Landeskrankenhausgesellschaft und Landesverbänden der Krankenkassen angerufen werden. Ist zwar der Nutzen hinreichend belegt, aber das InEK vergibt nicht Status 1, darf das Medizinprodukt wie zuvor angewandt werden, ohne dass dafür eine gesonderte Erstattung besteht.

Weist die Methode das Potential für eine Behandlungsalternative auf, deren Nutzen aber nicht hinreichend belegt ist, kann ein Erprobungsverfahren nach §137e SGB V eingeleitet werden, in dessen Rahmen die jeweilige Methode temporär erstattungsfähig ist. Für die Erprobung legt der G-BA Anforderungen an die Durchführung, wissenschaftliche Begleitung und Auswertung sowie an die Qualität der Leistungserbringung fest. In der Kombination aus Nutzenbewertung und Erprobungsregelung handelt es sich ausschließlich um Methoden, die maßgeblich auf der Anwendung eines Medizinprodukts beruhen. Über diese darf der G-BA gemäß § 137e SGB V nur dann einen Beschluss zur Erprobung fassen, wenn der Medizinproduktehersteller im Vorfeld zustimmt, die entstehenden Kosten für die wissenschaftliche Begleitung und Auswertung zu übernehmen. An der Erprobung teilnehmen können alle Leistungserbringer, die dem G-BA gegenüber mitteilen, die festgelegten Qualitätskriterien zu erfüllen. Eine dauerhafte Entscheidung soll in der Regel innerhalb von zwei Jahren getroffen werden (für eine ausführliche Einführung zur Erprobungsregelung siehe Olberg et al. 2014 ${ }^{31}$ ).

Kommt der G-BA zu dem Schluss, dass die Methode schädlich oder unwirksam ist, wird die Anwendung der Methode aus der Leistungserbringung in Deutschland ausgeschlossen. Dies betrifft nicht nur die Leistungserbringung mit kollektiver Erstattung auf Grundlage von DRGs, sondern jegliche Anwendung und Erstattung der Methode, zum Beispiel im Rahmen von selektivvertraglichen Regelungen. 


\section{Nutzenbewertung von Methoden mit Medizinprodukten hoher Risikoklassen (§ 137h SGB V)}

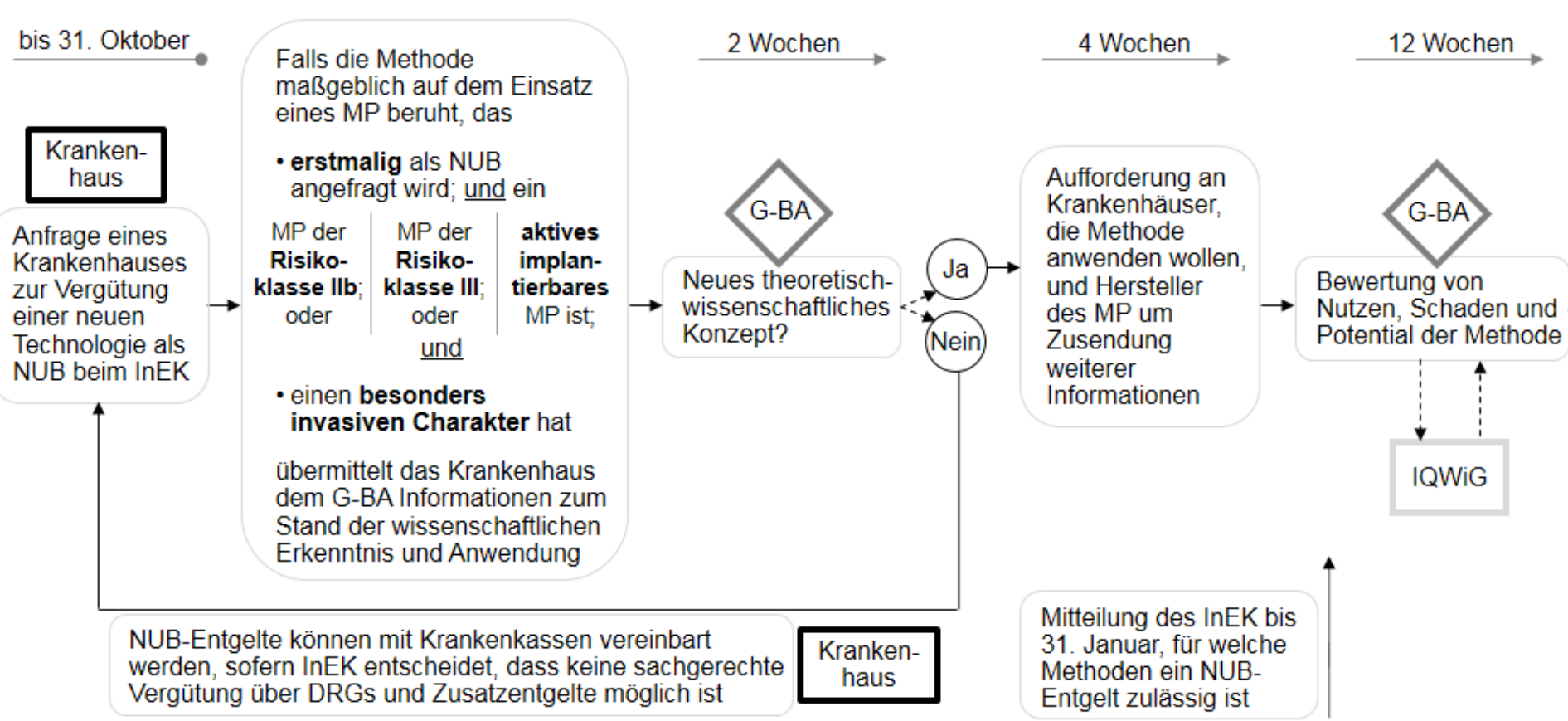

1. Nutzen hinreichend belegt

- Methode darf angewandt werden

- Falls Abrechnung nicht über DRGs möglich ist

- Vereinbarung eines Entgeltes innerhalb von 3 Monaten

- Qualitätskriterien der Leistungserbringer zu regeln?

2. Nutzen nicht hinreichend belegt, aber Potential

- Entscheidung über Erprobung (nach § $137 \mathrm{e}$ SGB V)

- Jedes Krankenhaus, das Methode anwenden möchte, muss an Studie teilnehmen

\section{Kein Potential}

- Methode ist von Leistungserbringung in Deutschland ausgeschlossen

\section{Legende}

- Beteiligung der Krankenhäuser

ㅁ Entscheidung durch den G-BA

Beauftragung des IQWiG möglich

\section{Abkürzungen}

DRG .....Diagnosis-Related Groups

G-BA.....Gemeinsamer Bundesausschuss

InEK ...... Institut für das Entgeltsystem im Krankenhaus
IQWiG ...... Institut für Qualität und Wirtschaftlichkeit im Gesundheitswesen MP......... Medizinprodukt

NUB........ Neue Untersuchungs- und Behandlungsmethode

eigene Darstellung; Quellen: Sozialgesetzbuch V § 137h und Medizinproduktemethodenbewertungsverordnung 


\section{Abschätzung der Tragweite der Regelung}

Die Beschreibung im vorigen Abschnitt legt dar, welche Kriterien erfüllt sein müssen, damit eine Bewertung des Nutzens durch den G-BA vorgenommen wird und anschließend eine Erstattung mit den Krankenkassen verhandelt werden darf: Für das Kriterium der "Erstattungsfähigkeit“ bedarf es der Bewertung durch das InEK mit "Status 1“. Für die Nutzenbewertung werden die Kriterien „Produktgruppe“ (Risikoklasse des Medizinproduktes und Invasivität) sowie „Neuheit“ (erstmalige Anfrage als NUB-Entgelt) herangezogen.

Um die Relevanz und den Umfang der Neuregelung einschätzen zu können, wird im Folgenden auf Basis der NUB-Aufstellung des Jahres 2016 analysiert, wie viele Medizinprodukte diese Kriterien tatsächlich erfüllen. Datenbasis ist die NUBAufstellung des InEK für das Jahr 2016. Dafür wurden zunächst alle NUBs einer Produktgruppe und - im Falle eines Medizinprodukts - einer Risikoklasse gemäß EU-Richtlinien auf Grundlage der vorhandenen Angaben zugeordnet. Mit der NUBAufstellung des Vorjahres wurde abgeglichen, ob die NUB erstmalig angefragt wurde. Abschließend wird konkret gezeigt, wie viele Medizinprodukte der NUBAufstellung 2016 alle Kriterien gleichzeitig erfüllt hätten und damit einer Nutzenbewertung unterzogen worden wären.

Kriterium „Erstattungsfähigkeit“ (Status 1)

Für das Jahr 2016 wurden insgesamt 30.080 Anfragen an das InEK gestellt, welche auf $710^{i}$ verschiedene NUBs verteilt waren. Von allen NUBs beruhen 505 Methoden maßgeblich auf dem Einsatz eines Medizinproduktes (71 Prozent). Insgesamt haben knapp 23 Prozent der 710 in 2016 angefragten NUBs vom InEK die Bestätigung erhalten, den Kriterien für ein NUB-Entgelt zu entsprechen und bislang nicht im DRG-Katalog abgebildet zu sein (Status 1). Für 21 dieser Anfragen gilt Status 1 nur in bestimmten Fällen." Bei alleiniger Betrachtung der 505 angefragten NUBs für Methoden mit Medizinprodukten beträgt der Anteil von Medizinprodukten mit Status 1 knapp 15 Prozent dieser Anfragen. (s. Tabelle 2). 
Tabelle 2: Ergebnisse der NUB-Anfragen für das Jahr 2016 nach Produktgruppe und Status

\begin{tabular}{|l|c|c|c|c|c|c|}
\hline & Status 1 & Status 2 & Status 3 & Status 4 & ausgesetzt & Total \\
\hline & $\begin{array}{c}\text { Kriterien } \\
\text { erfüllt }\end{array}$ & $\begin{array}{c}\text { Kriterien } \\
\text { nicht erfüllt }\end{array}$ & $\begin{array}{c}\text { Bearbeitung } \\
\text { nicht fertig }\end{array}$ & $\begin{array}{c}\text { Informa- } \\
\text { tionen } \\
\text { unplausibel }\end{array}$ & $\begin{array}{c}\text { G-BA } \\
\text { Beschluss } \\
\text { steht aus }\end{array}$ & \\
\hline Medizinprodukt & $75(15 \%)$ & $399(79 \%)$ & 0 & $28(6 \%)$ & 3 & 505 \\
\hline Arzneimittel & $85(41 \%)$ & $109(53 \%)$ & 0 & $11(5 \%)$ & 0 & 205 \\
\hline insgesamt & $160(23 \%)$ & $508(72 \%)$ & 0 & $39(5 \%)$ & 3 & 710 \\
\hline
\end{tabular}

eigene Darstellung; Quelle: NUB-Aufstellung des InEK 2016

\section{Kriterium Produktgruppe}

Bezogen auf alle neuen Medizinprodukte wird angenommen, dass rund zwei Prozent der Klasse III ${ }^{17,32}$ und neun Prozent der Klasse llb $^{32}$ zugeordnet sind. In der NUBAufstellung sind knapp 70 Prozent aller Methoden, die auf der Anwendung eines Medizinproduktes beruhen, diesen Risikoklassen zuzuordnen. Hinzu kommen knapp vier Prozent für aktive implantierbare Geräte. Diese große Differenz beim Anteil risikoreicher Produkte ist wahrscheinlich zum einen darauf zurückzuführen, dass vorrangig komplexere Verfahren und risikoreichere Produkte teurere Medizinprodukte sind, die eine zusätzliche Erstattung benötigen. Darüber hinaus ist davon auszugehen, dass insbesondere in der ambulanten Versorgung eine Vielzahl risikoarmer Medizinprodukte z. B. als Hilfsmittel Anwendung findet.

Die zusätzliche Anforderung eines „besonders invasiven Charakters“ grenzt diese Gruppe ein - je nach Kategorie jedoch unterschiedlich stark (s. Abbildung 2). Aktive implantierbare Geräte gelten hierbei grundsätzlich als besonders invasiv. ${ }^{28}$ Die Zuordnung eines Medizinproduktes zu Risikoklasse III ist häufig auf dessen spezielle Anwendung in direktem Kontakt mit Herz, Kreislauf oder Nervensystem zurückzuführen. Die Anforderung für einen besonders invasiven Charakter - diese Organe bzw. Organfunktionen langzeitig zu verändern oder zu ersetzen - ist dann eine relativ häufig erreichte Hürde. Die Gruppe der Medizinprodukte llb ist insgesamt heterogener als die der Klasse III. Im Vergleich zu Klasse III ist demnach die Anforderung eines besonders invasiven Charakters in Klasse llb, nämlich Energie oder radioaktive Stoffe auszusenden, bei einem geringeren Anteil der Medizinprodukte erfültt. 


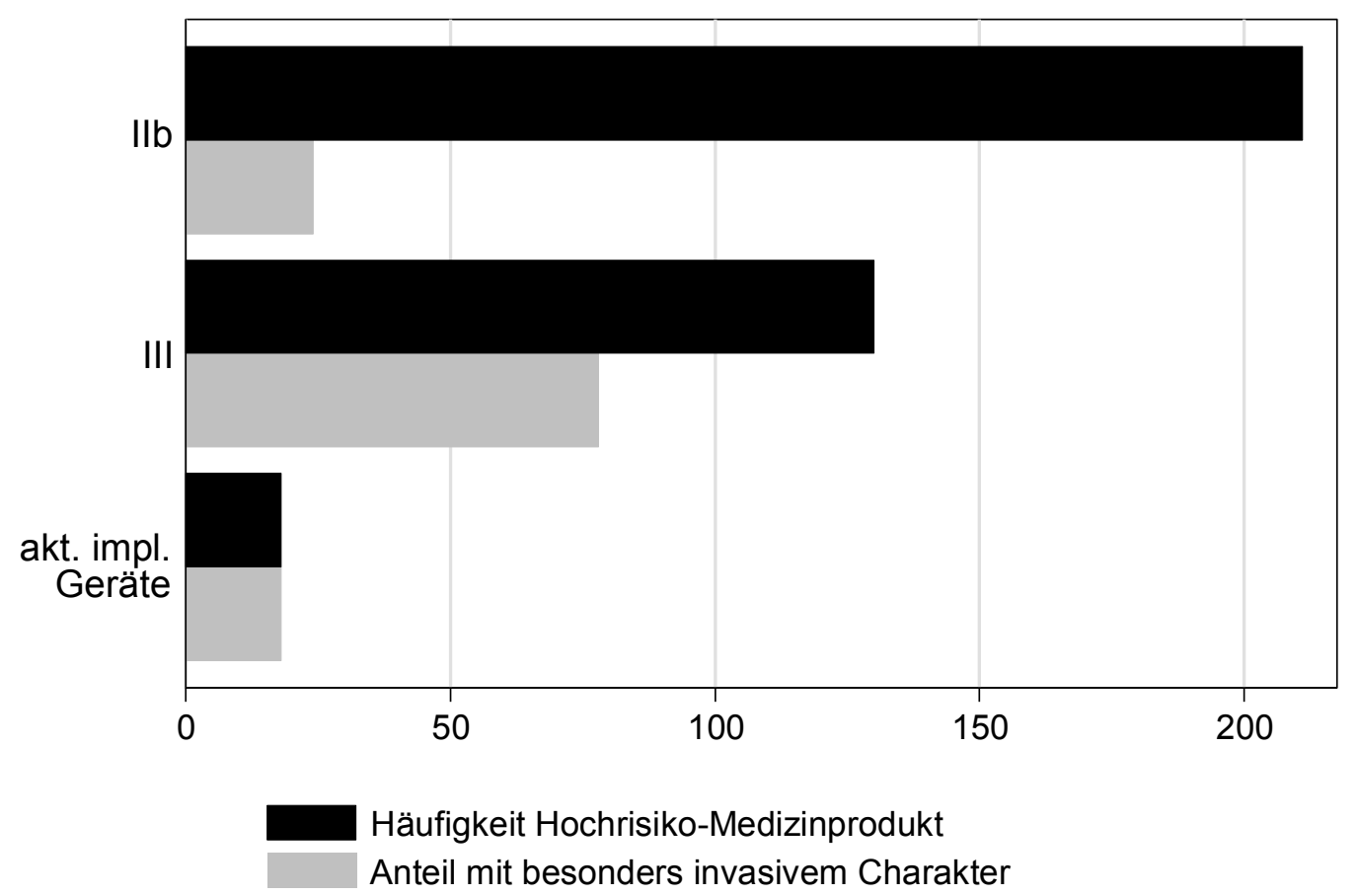

eigene Darstellung und Zuordnung der Risikogruppen; Quelle: NUB-Aufstellung des InEK 2016

Zusätzlich hat der G-BA zu prüfen, ob es sich bei den Fällen um ein neues theoretisch-wissenschaftliches Konzept handelt. Damit wird festgelegt, dass prinzipiell nur Wirkmechanismen und nicht einzelne Medizinprodukte bewertet werden. In der Rechtsverordnung ist explizit festgelegt, dass eine schrittweise Weiterentwicklung, sogenannte „Schrittinnovationen“, davon nicht betroffen ist. Eine quantitative Beurteilung ist auf der vorhandenen Datengrundlage und ohne die individuelle Entscheidung des G-BA nicht plausibel.

\section{Kriterium „Neuheit“ (erstmalige Anfrage)}

Krankenhäuser können für Methoden - unabhängig von deren Status - in darauffolgenden Jahren erneut eine NUB-Anfrage stellen. Von den 505 Medizinprodukten sind 87 (17\%) nicht in der NUB-Aufstellung des Vorjahres gelistet, sodass es sich in diesen Fällen um eine „erstmalige Anfrage“ handelt.

Allerdings ist das Ergebnis des NUB-Status bei erneuter Anfrage nicht immer identisch. Von den Medizinprodukten hat sich bei vier Produkten der Status im Vergleich zu 2015 geändert: ein Medizinprodukt hatte 2015 Status 1, 2016 aber Status 2; zwei erhielten 2015 Status 2, aber 2016 Status 1 und ein Medizinprodukt wurde von Status 2 auf Status 4 geändert. Der Wechsel des Status ist bei 
Arzneimitteln im aktuellen Jahr deutlich häufiger. Dort haben von 2015 zu 2016 knapp 10 Prozent ihren NUB-Status verändert. Diese Statuswechsel sind insofern für die Nutzenbewertung relevant, als dass die Wahrscheinlichkeit, Status 1 zu erhalten, mit erneuter Anfrage steigt. Von allen Medizinprodukten, die 2016 erstmalig als NUB angefragt wurden, haben knapp sechs Prozent Status 1 zugesprochen bekommen, jedoch knapp 17 Prozent der Medizinprodukte mit erneuter Anfrage. (s. Abbildung 3) Für die Nutzenbewertung werden aber nur jene Medizinprodukte berücksichtigt, die bereits im ersten Antragsjahr Status 1 erhalten. Es ist zu hoffen, dass dadurch nicht ein Anreiz geschaffen wird, im ersten Jahr eine sehr spärliche Anfrage abzugeben, um dadurch eine Nutzenbewertung bei „erneuter“ Anfrage zu umgehen.

Abbildung 3: Verteilung der NUB-Status von Medizinprodukten bei erstmaliger und erneuter Anfrage

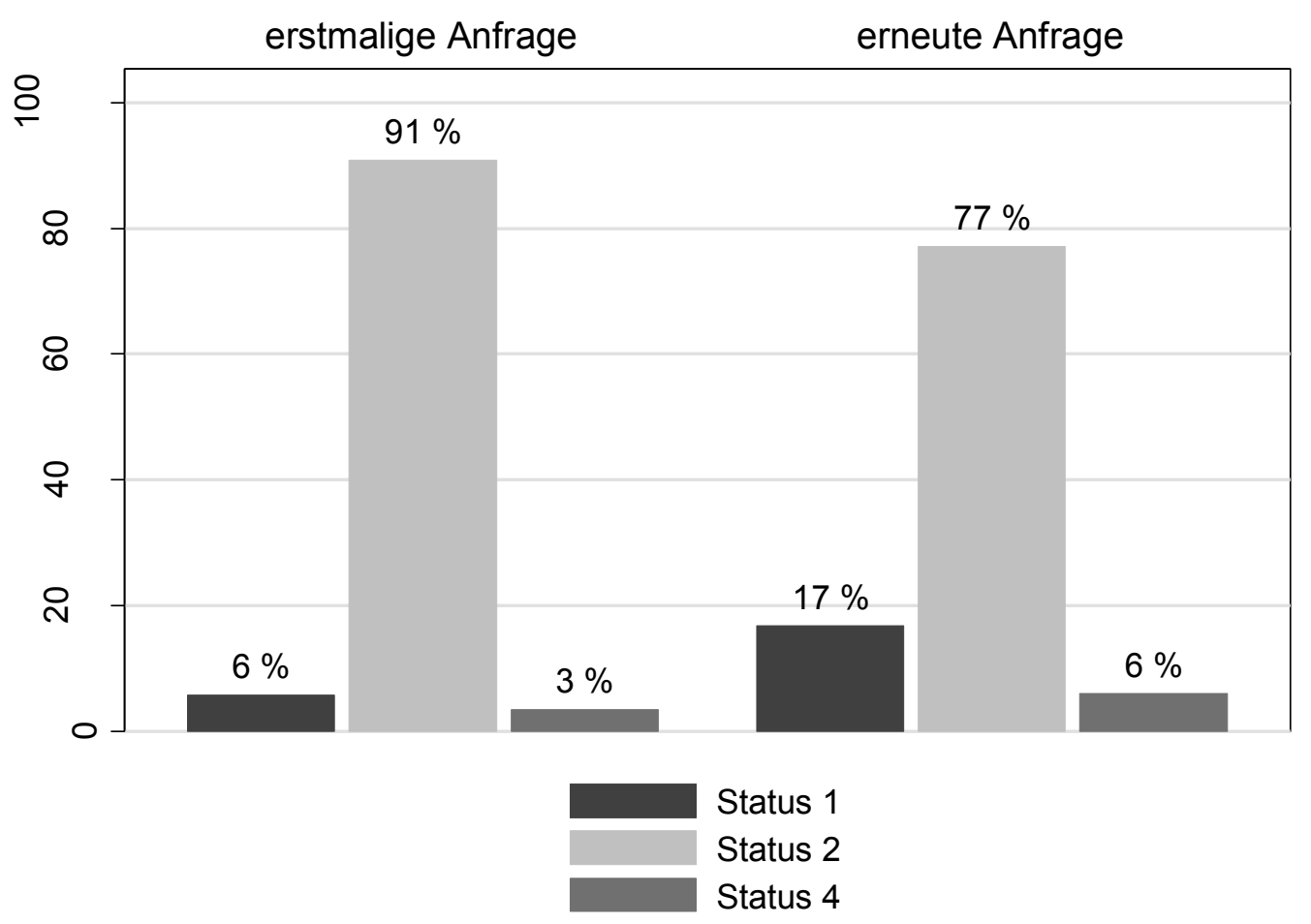

eigene Darstellung; Quelle: NUB-Aufstellung des InEK 2016 und 2015

Insgesamt wird durch die Diskussion deutlich, dass der Umfang der in die Regelung eingeschlossenen Hochrisiko-Medizinprodukte gering ist. Von den Medizinprodukten 2016 erfüllen auf Grundlage der vorgenommenen Zuordnung zu Risikoklassen exakt drei Medizinprodukte die Anforderungen hinsichtlich Produktgruppe, 
Erstattungsfähigkeit und Neuheit. Dabei ist noch nicht geprüft, ob ein neues theoretisch-wissenschaftliches Konzept vorliegt.

Dabei ist anzumerken, dass der G-BA aufgrund des Ablaufs des Verfahrens deutlich mehr Verfahren prüfen wird, als überhaupt für eine Vergütung infrage kommen. Erst ungefähr sechs Wochen vor Ablauf der Bewertungsphase veröffentlicht das InEK das Ergebnis darüber, welche NUBs nicht im DRG-System abgebildet sind, für die ein Entgelt verhandelt werden darf. Ignoriert man daher den NUB-Status, so erfüllen 31 Hochrisiko-Medizinprodukte die Kriterien zu einer Prüfung.

\section{Fazit}

Die Einführung einer systematischen Bewertung des Nutzens neuer ärztlicher Methoden ist im Sinne der Patientensicherheit und für die Überprüfung der patientenrelevanten Wirksamkeit unter Alltagsbedingungen eine positive Ergänzung zum bestehenden System. Allerdings zeigt die Auswertung der NUBs aus dem Jahr 2016, dass einige Aspekte der Regelung zu hinterfragen sind.

Abgesehen von den inhaltlichen Einschränkungen (erstmalige Anfrage für ein NUBEntgelt, hohe Risikoklasse, besonders invasiver Charakter, neues theoretischwissenschaftliches Konzept) betrifft die Regelung aufgrund der Systematik der DRGVergütung prinzipiell teurere Methoden. Kann eine neue Methode mit risikoreichem Medizinprodukt für den Preis einer bestehenden DRG-Fallpauschale erbracht werden, gelangt die Methode weiterhin ungeprüft in die Versorgung. Damit ist die Nutzenbewertung als an das NUB-Entgelt gekoppeltes Verfahren in erster Linie ein Instrument im Rahmen der Vergütungsfindung, weniger ein Sicherheitsinstrument. Aus Systemsicht könnten die erstmaligen Durchführungen von Nutzenbewertungen auch zunächst nur eine Handvoll pro Jahr - mittelfristig durchaus einen Einstieg in weitere Nutzenbewertungen im stationären Sektor bedeuten. So ist denkbar, dass zukünftig z. B. auch neue Methoden mit Medizinprodukten hoher Risikoklassen, für die kein NUB-Entgelt beantragt wird, oder auch Arzneimittel, für die ein NUB-Entgelt beantragt wird, einer Nutzenbewertung im stationären Sektor unterzogen werden.

Bis dahin müssen aber für die anstehenden Nutzenbewertungen nicht nur methodische Fragen geklärt werden - sondern auch Fragen der Akzeptanz. So bestehen für Hersteller geringe Anreize aber hohe Risiken, die Nutzenbewertung zu 
durchlaufen: Während des Verfahrens erfolgt keine automatische Vergütung. Selbst bei einem hinreichend belegten Nutzen durch den G-BA und Status 1 vom InEK gelten NUB-Entgelte nur für ein Jahr. Darüber hinaus wurde seit Einführung der Erprobungsregelung im Jahr 2012 keine Studie durchgeführt. Daher ist die Frage, ob im Zusammenhang mit der Nutzenbewertung dieses Verfahren zum Einsatz kommt. Eine kleine Verbesserung aus Sicht der Hersteller ist, dass bei belegtem Nutzen Krankenkassen einer Vergütung zustimmen müssen, was im allgemeinen NUBVerfahren nicht der Fall ist. Risiko des Verfahrens ist hingegen ein genereller Ausschluss der Leistungserbringung in Deutschland bei nicht festgestelltem Potential. Damit könnte bei Methoden oder Medizinprodukten ohne ausreichende Datenbasis bereits im Vorfeld strategisch eine alternative Vergütungsmöglichkeiten, z. B. durch Selektivverträge, oder ein niedrigerer Preis durch bestehende DRGs akzeptiert werden, um die Nutzenbewertung bewusst zu umgehen. Dabei ist zu berücksichtigen, dass NUB-Entgelte meist von Krankenhäusern initiiert werden - die damit z. T. andere Zielsetzungen wie etwa den Ausweis von Kompetenz und/oder Innovationsfreudigkeit in bestimmten Feldern verfolgen - und es daher nicht allein eine strategische Entscheidung von Herstellern ist, die Nutzenbewertung zu durchlaufen. Allerdings ist das Verfahren für Krankenhäuser sehr komplex und aufwendig, während keine Konsequenzen drohen und es kaum überprüfbar ist, wenn keine Mitteilung an den G-BA erfolgt. 


\section{Literatur}

1. Bundesministerium für Bildung und Forschung. Bildung und Forschung in Zahlen 2015. Hannover; 2015.

2. Lenkungskreis Nationaler Strategieprozess. Innovationen in der Medizintechnik: Eine gemeinsame Initiative des Bundesministeriums für Bildung und Forschung, des Bundesministeriums für Gesundheit und des Bundesministeriums für Wirtschaft und Technologie. Berlin; 2012.

3. Neumann U, Hagen, Anja, Schönermark, Matthias. Regulation der Aufnahme von innovativen nichtmedikamentösen Technologien in den Leistungskatalog solidarisch finanzierter Kostenträger; 2007.

4. Henschke C, Panteli D, Perleth M, Busse R. Taxonomy of Medical Devices in the Logic of Health Technology Assessment. International journal of technology assessment in health care 2015;31(5):324-30.

5. Sorenson C, Drummond M, Kristensen FB, Busse R. Wie kann die Gesundheitstechnologiebewertung wirkungsvoller gestaltet werden? Kopenhagen: Weltgesundheitsorganisation; 2008.

6. Lühmann D, Rüther A, Schwarzer R, Gawlik C, Schiffner R, Perleth M. Grundlagen und Prinzipien von Health Technology Assessment (HTA). In: Perleth M, Busse R, (eds). Health technology assessment: Konzepte, Methoden, Praxis für Wissenschaft und Entscheidungsfindung, 2nd ed. Berlin: MWV Med. Wiss. Verl.-Ges; 2014, p. 1-57.

7. Office of Technology Assessment. Policy Implications of the Computer Tomography (CT) Scanner: November 1978. Available at: http://ota.fas.org/reports/7813.pdf [accessed 16.03.2016].

8. Bundesausschuss der Ärzte und Krankenkassen. Richtlinien des Bundesausschuss der Ärzte und Krankenkassen über die Einführung neuer Untersuchungs- und Behandlungsmethoden: NUB-Richtlinien. Available at: https://www.g-ba.de/downloads/62-492-192/RL_NUB-1990-12-04.pdf [accessed 14.03.2016].

9. Busse R, Schreyögg J, Stargardt T. Management im Gesundheitswesen: Das Lehrbuch für Studium und Praxis. 3rd ed. Berlin, Heidelberg: Springer; 2013.

10.Zentner A, Busse R. Internationale Standards der Kosten-Nutzen-Bewertung. Gesundh ökon Qual manag 2006;11(6):368-73. 
11. European Network for Health Technology Assessment. Joint Action on HTA 20122015: HTA Core Model. Version 3.0; 2016.

12. Gerhardus A, Droste S, Engelke K, Gibis B, Hoffmann C, Lühmann D, Perleth M, Stich AK, Garrido MV, Zentner A. Bewertung der organisatorischen, rechtlichen, ethischen und soziokulturellen Aspekte von Technologien. In: Perleth M, Busse R, (eds). Health technology assessment: Konzepte, Methoden, Praxis für Wissenschaft und Entscheidungsfindung, 2nd ed. Berlin: MWV Med. Wiss. Verl.Ges; 2014, p. 263-320.

13.Windeler J. Nutzen und Nutzenbewertung. Deutsche medizinische Wochenschrift (1946) 2006;131(19 Suppl 1):S12-5.

14.Perleth M, Matthias K, Nocon M. Ermittlung von Wirksamkeit, Nutzen und Sicherheit einer Technologie. In: Perleth M, Busse R, (eds). Health technology assessment: Konzepte, Methoden, Praxis für Wissenschaft und Entscheidungsfindung, 2nd ed. Berlin: MWV Med. Wiss. Verl.-Ges; 2014, p. 209262.

15. Windeler J, Lange S. Nutzenbewertung medizinischer Leistungen im deutschen Gesundheitswesen - rechtlicher Rahmen, historische und internationale Perspektive. Bundesgesundheitsbl. 2015;58(3):220-6.

16. Lelgemann M, Most-Ehrlein S, Pazhur RJ. Medizinprodukte im Krankenhaus. In: Klauber J, Brenner G, Augurzky B, (eds). Mengendynamik: mehr Menge, mehr Nutzen? Stuttgart: Schattauer; 2013, p. 281-294.

17.Windeler J, Sauerland S, Ernst A. Warum Medizinprodukte in Deutschland einer besseren Regulierung bedürfen. In: Repschläger U, (ed). BARMER Gesundheitswesen aktuell 2011. Wuppertal: Barmer GEK; 2011, p. 152-166.

18.GKV-Spitzenverband. Medizinprodukte - Mythen und Wahrheit: Gemeinsames Argumentationspapier von den Spitzenverbänden der gesetzlichen Krankenkassen in Deutschland. Berlin; 2013.

19.Zens Y, Fujita-Rohwerder N, Windeler J. Nutzenbewertung von Medizinprodukten. Bundesgesundheitsblatt, Gesundheitsforschung, Gesundheitsschutz 2015;58(3):240-7.

20. Busse R, Stargardt T, Schreyögg J. Determining the "Health Benefit Basket" of the Statutory Health Insurance scheme in Germany: methodologies and criteria. The European journal of health economics HEPAC health economics in prevention and care 2005;Suppl:30-6. 
21. Deutscher Ethikrat. Nutzen und Kosten im Gesundheitswesen - Zur normativen Funktion ihrer Bewertung. Berlin; 2011.

22. Sachverständigenrat zur Begutachtung der Entwicklung im Gesundheitswesen. Bedarfsgerechte Versorgung: Perspektiven für ländliche Regionen und ausgewählte Leistungsbereiche; Gutachten 2014. 1st ed. Bern: Huber; 2014.

23.GKV-Spitzenverband. Neue Untersuchungs- und Behandlungsmethoden (NUB). Available at: https://www.gkvspitzenverband.de/krankenversicherung/krankenhaeuser/drg_system/neue_unter suchungs_und_behandlungsmethoden_nub/neue_untersuchungs_und_behandlu ngsmethoden_nub.jsp; 2016 [accessed 09.02.2016].

24. Levaggi R, Moretto M, Pertile P. Two-part payments for the reimbursement of investments in health technologies. Health policy (Amsterdam, Netherlands) 2014;115(2-3):230-6.

25.Scheller-Kreinsen D, Quentin W, Busse R. DRG-based hospital payment systems and technological innovation in 12 European countries. Value in health the journal of the International Society for Pharmacoeconomics and Outcomes Research 2011;14(8):1166-72.

26. Institut für das Entgeltsystem im Krankenhaus (InEK). Verfahrenseckpunkte: Anfragen nach § 6 Abs. 2 KHEntgG (Neue Untersuchungs- und Behandlungsmethoden). Available at: http://www.gdrg.de/cms/Neue_Untersuchungs_und_Behandlungsmethoden_NUB/Verfahrenseckpunkte; 2015 [accessed 10.03.2016].

27. Bundessozialgericht. B 1 KR 19/96 R; 1998.

28. Bundesministerium für Gesundheit. Verordnung über die Voraussetzungen für die Bewertung neuer Untersuchungs- und Behandlungsmethoden mit Medizinprodukten hoher Risikoklasse nach § 137h des Fünften Buches Sozialgesetzbuch (Medizinproduktemethodenbewertungsverordnung - MeMBV). 29.Europäisches Parlament und Rat. Richtlinie 93/42/EWG des Rates vom 14. Juni 1993 über Medizinprodukte: 1993 L0042.

30. Europäisches Parlament und Rat. Richtlinie des Rates vom 20. Juni 1990 zur Angleihung der Rechtsvorschriften der Mitgliestaaten über aktive implantierbare medizinische Geräte (90/385/EWG): 1990 L0385. 
31. Olberg $B$, Perleth $M$, Busse $R$. The new regulation to investigate potentially beneficial diagnostic and therapeutic methods in Germany: Up to international standard? Health Policy 2014;117(2):135-45.

32. Winkler O. Innovation, Sicherheit und Nutzen von Medizinprodukten - Sicht der Industrie. Available at: http://www.netzwerkversorgungsforschung.de/uploads/Winkler,Olaf\%20Innovation,\%20Sicherheit\%20 und\%20Nutzen\%20unter\%20ge\%C3\%A4nderten\%20regulatorischen\%20Rahme nbedingungen.pdf; 2013 [accessed 07.03.2016].

\footnotetext{
' Bei drei NUBs steht eine Entscheidung des G-BA aus, sodass deren Bearbeitung beim InEK bis zum G-BA Beschluss ausgesetzt ist. Sie sind nicht in den folgenden Berechnungen enthalten.

ii In Einzelfällen vergibt das InEK zwei Status, die jeweils in unterschiedlichen Fällen gelten. In der Übersicht sind alle in ihrer "bestmöglichen“ Kategorie (Status 1 oder Status 4) zugeordnet.
} 